\title{
Initial Factors Predicting Functional Performance in Patients with Traumatic Tetraplegia
}

\author{
P. Daverat, ${ }^{1}$ J. F. Dartigues, ${ }^{2}$ J. M. Mazaux, ${ }^{1}$ M. Barat ${ }^{1}$ \\ ${ }^{1}$ Service Rééducation Fonctionnelle Neurologique, Tastet Girard Hôpital Pellegrin, \\ 33076 Bordeaux Cedex, France, ${ }^{2}$ Laboratoire d'Epidémiologie, Biostatistique, Infor- \\ matique Médicale Université de Bordeaux II, 33076 Bordeaux Cedex, France.
}

\section{Summary}

The authors present a prospective analysis of the functional outcome in 99 patients with traumatic tetraplegia consecutively admitted to the Pellegrin Hospital (University Hospital, Bordeaux, France). There was a 29\% death rate. Eighteen months after injury, 33\% of the patients were dependent (not able to perform activities of daily living without the presence of a helping individual), 38\% were independent. Two predictors of independence (age and initial Yale Scale Score) were found by a statistical analysis of the course of the disorder.

Key words: Spinal cord injury; Functional performance; Prognosis.

With improvement of the management of spinal cord injury patients (SCI), including the early transfer to a specialised unit, the intensive care facilities available and the involvement of a full range of specialist services, patients with traumatic tetraplegia can anticipate an increasingly improved prognosis. Nevertheless, reported rates remain variable concerning the ability to predict accurately, even amongst patients with similar injury, for those who will obtain an optimal functional outcome. The present study concerns 99 patients with traumatic tetraplegia, admitted to the emergency room of Pellegrin Hospital (University Hospital, Bordeaux). The major objective was to estimate the outcome of these patients and to determine initial predictors of functional performance.

\section{Patients and methods}

From January 1982 to December 1985, we included 99 patients with traumatic tetraplegia, initially and consecutively admitted to the emergency ward of Pellegrin Hospital (Bordeaux, France). Within 6 hours after admission, our rehabilitation team systematically collected data on their initial neurological state. Each subject was followed up monthly by one member of the team for a minimum period of 1 year.

The outcome was studied as a function of time in a longitudinal prospective 
analysis. When survival is assured, reintegration to normal living patterns can be viewed as the ultimate goal. Most professionals wish each patient to live his life as normally as possible, within the constraints of the aftermath of injury. In this sense, 'living' obviously involves functional performance.

Many techniques measuring function have been reported in the literature in the past decade. The most common indicators are those which measure basic self-care routines such as feeding, dressing, toileting, transferring and moving. Collectively, these are most often referred to as activities of daily living (ADL), and are exemplified by such studies as the Katz index of ADL (Katz et al., 1963), the Barthel index (Mahoney and Barthel, 1965), the Kenny Self-Care Evaluation (Schoening et al., 1965).

In the present study on traumatic tetraplegia, when survival was assured, we selected two functional states: dependence or independence. A patient with traumatic tetraplegia was defined as independent if he could perform basic selfcare activities such as feeding, dressing, washing, toileting, transferring and moving (in wheelchair or walking) without the presence of a helping individual, although he might need assistive devices. In this sense, the status of independence corresponded to a Barthel index of more than 60 .

Several potential predictors of independence were selected for the initial examination. These included age, level of lesion, Yale Scale Score (YSS) (Chehrazi et al., 1981) initial conscious level. YSS allows grading of the lesional and sublesional neurological findings. It is a quantitative measurement which does not have the ambiguous characters of the scales generally used (Frankel et al., 1969; Maynard et al., 1979). The score is determined by motor and sensory testing, each on a scale of 5 points. The total score varies from 0 to $10 ; 0$ indicates a complete lesion with absence of motor and sensory function below the level of injury, while 10 corresponds to intact medullary function. This scoring system is helpful since it is simple and reliable and can easily be administered in the emergency ward.

The Markov model (Commenges et al., 1984) was used to estimate the probability of the outcome in 3 states as a function of time. Univariate analysis was used to estimate the probability of independence (Kaplan and Meier, 1958). Multivariate analysis (Cox, 1970, 1972) was also used to determine predictors of independence in traumatic tetraplegia.

\section{Results}

The total sample consisted of 99 patients with traumatic tetraplegia, $78 \%$ were males, with a male:female ratio of $4: 1$. During the first month, 3 subjects were lost to follow-up while another 2 were lost in the following 6 months. The subjects ranged in age from 12 to 84 years (mean age $=41$ years). A peak of $35 \%$ was found in the range 20 to 30 years. Upon admission, $12 \%$ of the patients were comatose and 59\% had normal consciousness. Forty seven per cent of the tetraplegics had a complete lesion (YSS $=0$ ). The mean YSS was 2 . Within this population, $25 \%$ were injured at the $\mathrm{C} 4$ level, $31 \%$ at the $\mathrm{C} 5$ level, $23 \%$ at the $\mathrm{C} 6$ level and $13 \%$ at the $\mathrm{C} 7$ level.

Figure 1 shows the outcome of the 99 patients in 3 states: death, dependence, independence. The overall mortality rate was $29 \%$ at 3 months. Eighteen months after injury, $33 \%$ of the patients were dependent in ADL, $38 \%$ were independent. 


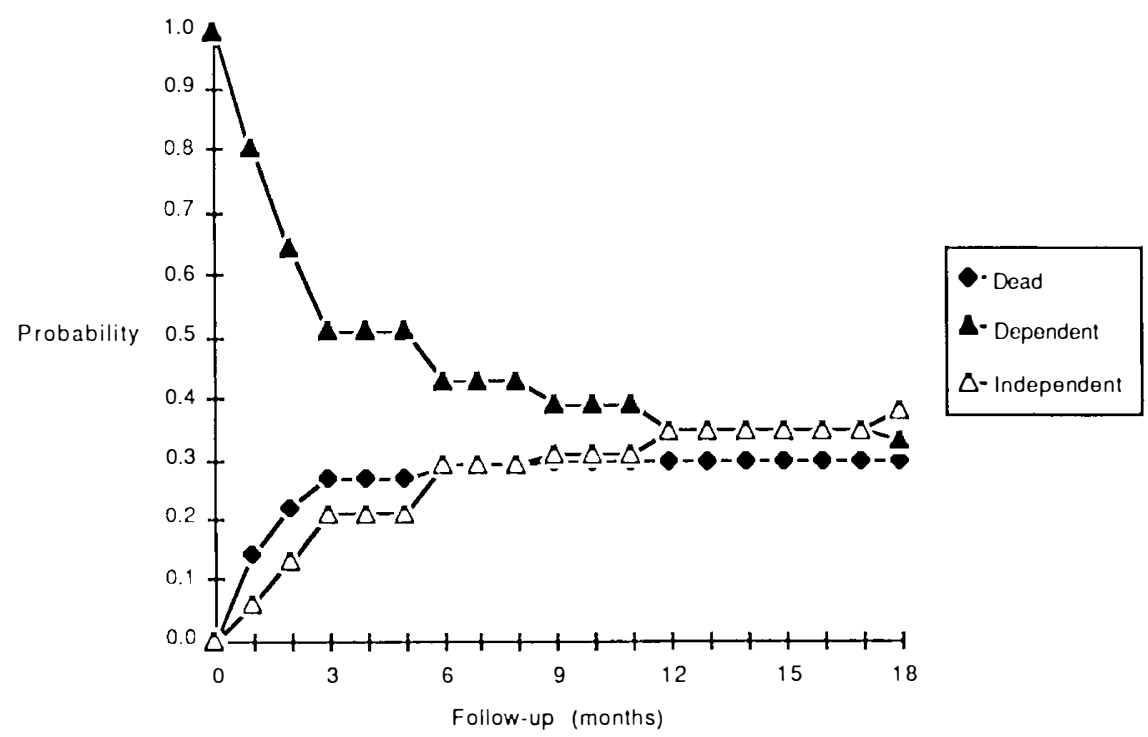

Figure 1 Outcome curve for the total sample (99 patients with traumatic tetraplegia).

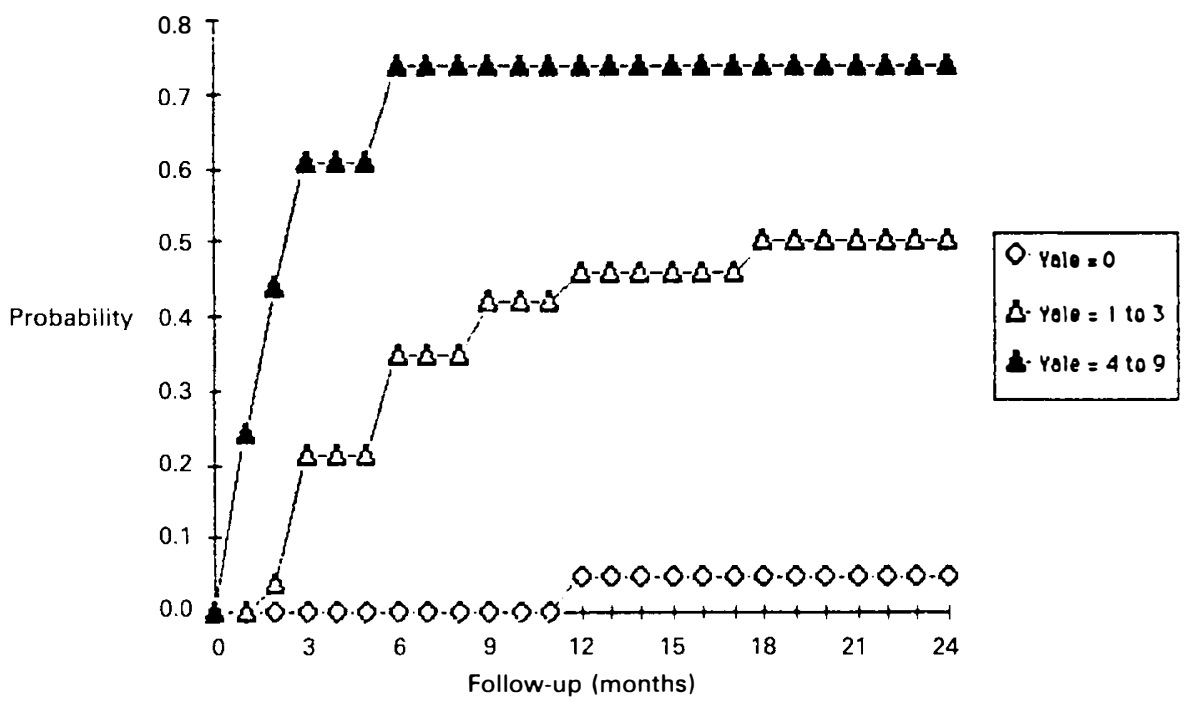

Figure 2 Independence curve as a function of initial YSS.

Figure 2 shows the independence curve of the total sample as a function of the initial YSS. YSS is a predictive factor of independence $(p<0.0001)$.

Figure 3 shows the independence curve as a function of the level of the lesion. The result is not significant $(\mathrm{p}=0 \cdot 41)$. Statistically, the level of the lesion is not a predictor of indepence in patients with traumatic tetraplegia.

With multivariate analysis, predictors of independence are age $(\mathrm{p}<0.001)$ and initial YSS $(p<0.00001)$. Initial conscious level $(p=0 \cdot 13)$ and lesion level $(\mathrm{p}=0.68)$ are not significantly correlated with independence. 


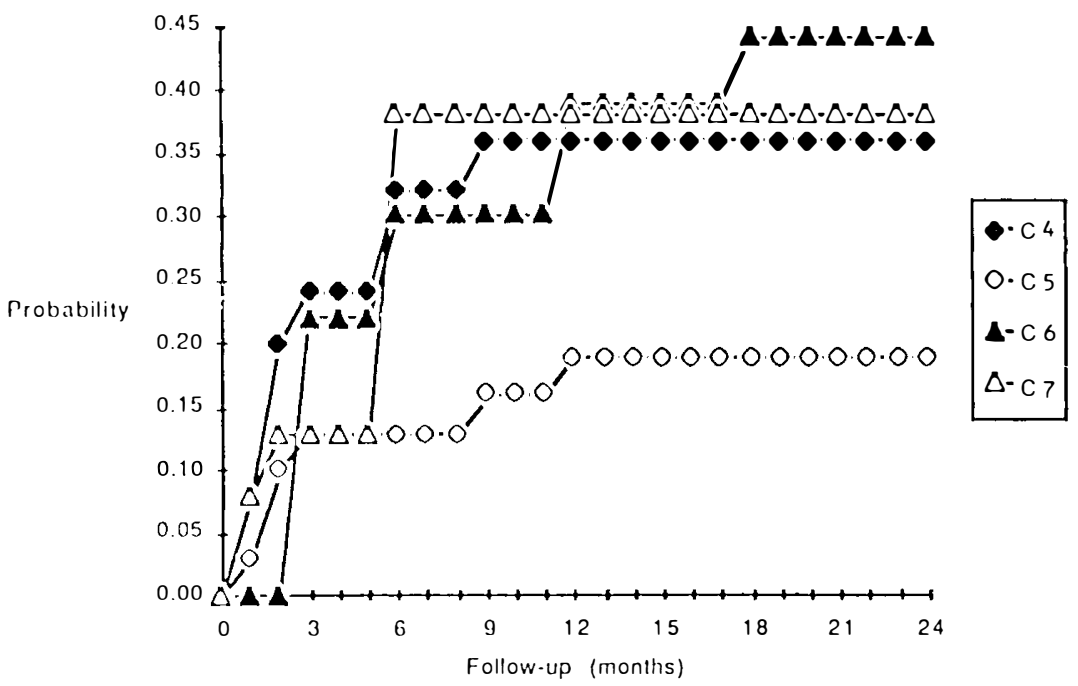

Figure 3 Independence curve as a function of lesion level.

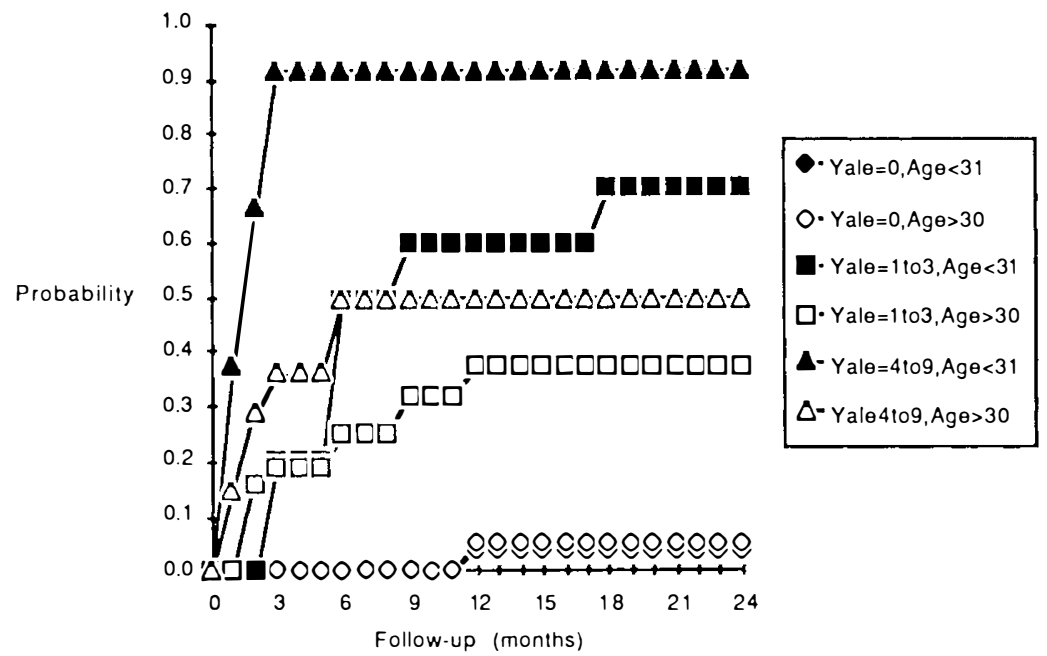

Figure 4 Predictive model of independence as a function of age and initial YSS.

Figure 4 shows a predictive model of independence in tetraplegic patients as a function of age and initial YSS.

\section{Discussion}

Among our tetraplegic patients, a peak was found in the age group 20 to 30 years. Fifty per cent of the sample was over 40 years, thus spinal cord injuries are not exclusively confined to young people (Harris et al. 1980). Forty seven per cent of the tetraplegic patients had a complete lesion; this is a reflection of our sample being of consecutive primary admissions. These results differ from previously 
published series (Silver and Gibbon, 1968) that showed the prevalence of incomplete lesions in tetraplegics, because such series were based on both primary and secondary admissions to rehabilitation centres. Upon admission, $12 \%$ of the patients were in a coma and $60 \%$ had normal consciousness. These results are in agreement with other major studies (Maynard et al., 1979; Harris et al., 1980).

To describe the outcome of the tetraplegic patients, we only selected 3 states: death, dependence, independence. The prognosis of mortality and walking recovery was already studied by the authors (Daverat et al., 1989). The present work focused on the notion of independence. In fact, tetraplegic patients who survived belong to two functional groups: they are dependent according to our definition (bed-confined or dependent in wheelchair) or they are not (independent in wheelchair or walking). Overall, on the day of the injury, our prediction showed that 3 almost equal states will distinguish tetraplegic patients: one third will die, another third will be dependent and a last third will be independent. Frankel et al. (1969) reported that $33 \%$ out of 218 cervical lesions are 'motor useful' after 1 year, and 10\% completely recover. Maynard et al. (1979) followed 123 patients. Thirty per cent are 'motor useful' and 10\% completely recover. These series published in 1969 and 1979 are not comparable to ours because they did not take into account patients who died during the first year following the injury. If we had used the same data in our sample, more than $55 \%$ of the patients should have become independent, which is high compared to the reports of Frankel and Maynard. Thus in 1989, when survival was assured, progress of medical care really improved the functional outcome of the spinal cord injured patients.

To predict the independence in tetraplegic patients, we used a uni- and multivariate analysis. With univariate analysis, YSS was a predictor of independence $(\mathrm{p}=0.0001)$. So the severity of the neurological injury is very significant, as it was for the walking status recovery (Daverat et al., 1988).

The lesion level is not significant $(p=0.68)$. This result could be explained because our independence evaluation is very rough. Lesion level could be important for particular activities such as prehension and wheelchair locomotion (Minaire, 1982) but not for global independence in ADL.

With multivariate analysis, two predictors of independence were found in our study: age and the initial YSS. Lesion level still remained insignificant. It could be explained by a selection bias: tetraplegic patients who died had the highest lesions (Daverat et al., 1989).

The predictive model (Fig. 4) shows that whatever their age, the patients with a complete lesion (YSS $=0$ ) will remain dependent. When the YSS is from 1 to 3 , age is an essential factor: under 30 years, $70 \%$ of the patients will be independent over 30 years, only $38 \%$. When the lesion is very incomplete (YSS $>4$ ), $90 \%$ of the subjects under 30 years will be independent, only $50 \%$ over 30 years.

\section{Conclusion}

From the present results, we can conclude that among the tetraplegic patients who are admitted to the hospital on the day of the injury, one third will die, another third will be dependent in the activities of daily living and the final third will be independent. Statistical analysis enabled us to isolate two predictors of independence: age and initial YSS. We can appreciate the importance of the YSS, which 
is a classification of acute spinal cord injury related more precisely to the neurological deficit. The patients are delineated soon after injury and we can appropriately manage and evaluate different therapeutic modalities.

\section{References}

Chehrazi B, Wagner F, Collins W, Freeman D 1981 A scale for evaluation of spinal cord injury. Fournal of Neurosurgery 54:310-315.

Commenges D, Barberger-Gateau P, Dartigues JF, Loiseau P, Salamon R 1984 A nonhomogeneous Markov chain model for follow-up studies with application to epilepsy. Methods of Information in Medicine 23:109-114.

Cox DR 1970 The Analysis of Binary Data. Chapman and Hall, London.

Cox DR 1972 Regression models of life tables. Fournal of the Statistical Society 34:187-220.

DAVERAT P, SibraC MC, DARTIGUES JF et al. 1988 Early prognostic factors for walking in spinal cord injuries. Paraplegia 26:255-261.

Daverat P, Gagnon M, Dartigues JF, Mazaux JM, Barat M 1989 Initial factors predicting survival in patients with spinal cord injury. Fournal of Neurology, Neurosurgery and Psychiatry. 52:403-406.

Frankel HL, HANCOCK DO, Hyslop G et al 1969 The value of postural reduction in the initial management of closed injuries of the spine with paraplegia and tetraplegia. Paraplegia 14:262275.

Harris P, Karmi MZ, Marclemont E, D'Albret M, Paul KS 1980 The prognosis of patients sustaining severe cervical spine injury (C2-C7 inclusive). Paraplegia 18:324-330.

KAPLAN EL, MEIER P 1958 Non parametric estimation from incomplete observations. Fournal of the American Statistical Association 53:457-481.

KaTZ S, Ford AB, Moskowitz RW, JaCKSON BA, JAFFE MW 1963 Studies of illness in aged: Index of ADL: standardized measure of biological and psychosocial function. FAMA 185:914-919.

MAHONEY FI, BARTHEL W 1965 Functional evaluation. Barthel Index Md. State Medical fournal $14: 61-65$.

Maynard FM, Reynolds GG, Fountain S, Wilmot C, Hamilton R 1970 Neurological prognosis after traumatic quadriplegia. Fournal of Neurosurgery 50:611-661.

Minaire P 1982 guide pratique de la rééducation et de la réadaptation. In: Masson (ed) Paraplégie et Tetraplégie, 2nd edn.

SCHOENing HA, ANDEREgG L, Bergstrom D et al. 1965 Numerical scoring of self-care status of patients. Achives of Physical Medicine and Rehabilitation 46:689-697.

SilVER JR, GibBON NO 1968 Prognosis in tetraplegia. British Medical fournal 4:79-83. 